Thin film versus paper-like reduced graphene oxide: Comparative study of structural, electrical, and thermoelectrical properties

Olena Okhay', Gil Gonçalves, Alexander Tkach, Catarina Dias, Joao Ventura, Manuel Fernando Ribeiro da Silva, Luís Miguel Valente Gonçalves, and Elby Titus

Citation: Journal of Applied Physics 120, 051706 (2016); doi: 10.1063/1.4958956

View online: http://dx.doi.org/10.1063/1.4958956

View Table of Contents: http://aip.scitation.org/toc/jap/120/5

Published by the American Institute of Physics

\section{Looking for a specific} instrument?

Easy access to the latest equipment. Shop the Physics Today Buyer's Guide.

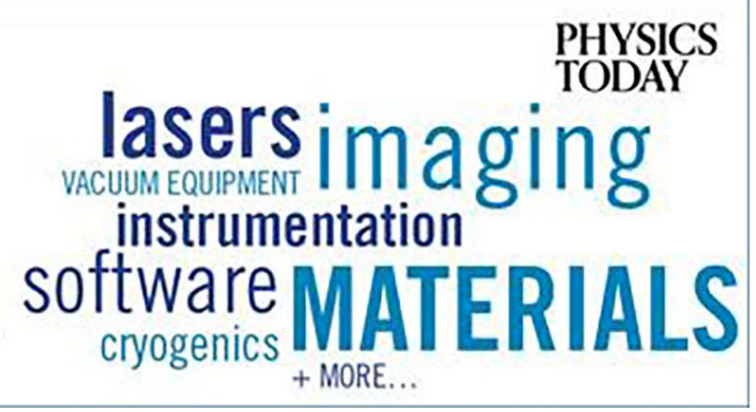




\title{
Thin film versus paper-like reduced graphene oxide: Comparative study of structural, electrical, and thermoelectrical properties
}

\author{
Olena Okhay, ${ }^{1, a)}$ Gil Gonçalves, ${ }^{1}$ Alexander Tkach, ${ }^{2}$ Catarina Dias, ${ }^{3}$ Joao Ventura ${ }^{3}$ \\ Manuel Fernando Ribeiro da Silva, ${ }^{4}$ Luís Miguel Valente Gonçalves, ${ }^{4}$ and Elby Titus ${ }^{1}$ \\ ${ }^{1}$ Nanotechnology Research Division, Center for Mechanical Technology and Automation (TEMA), \\ Department of Mechanical Engineering, University of Aveiro, 3810193 Aveiro, Portugal \\ ${ }^{2}$ CICECO Aveiro Institute of Materials, Department of Materials and Ceramic Engineering, \\ University of Aveiro, 3810193 Aveiro, Portugal \\ ${ }^{3}$ Institute of Physics of Materials of the University of Porto (IFIMUP), 4169007 Porto, Portugal \\ ${ }^{4}$ Industrial Electronics Department, University of Minho, Azurem, 4800058 Guimaraes, Portugal
}

(Received 3 November 2015; accepted 1 April 2016; published online 20 July 2016)

\begin{abstract}
We report fabrication of reduced graphene oxide ( $\mathrm{rGO}$ ) films using chemical reduction by hydrazine hydrate and rGO paper-like samples using low temperature treatment reduction. Structural analysis confirms the formation of the rGO structure for both samples. Current-voltage (I V) measurements of the rGO film reveal semiconductor behavior with the maximum current value of $\sim 3 \times 10{ }^{4} \mathrm{~A}$. The current for the rGO paper sample is found to be, at least, one order of magnitude higher. Moreover, bipolar resistance switching, corresponding to memristive behavior of type II, is observed in the I V data of the rGO paper. Although precise values of the rGO film conductivity and the Seebeck coefficient could not be measured, rGO paper shows an electrical conductivity of $6.7 \times 10^{2} \mathrm{~S} / \mathrm{m}$ and Seebeck coefficient of $-6 \mu \mathrm{V} /{ }^{\circ} \mathrm{C}$. Thus, we demonstrate a simplified way for the fabrication of rGO paper that possesses better and easier measurable macroscopic electrical properties than that of rGO thin film. Published by AIP Publishing. [http://dx.doi.org/10.1063/1.4958956]
\end{abstract}

\section{INTRODUCTION}

Graphene, a two-dimensional nanocarbon material, has attracted significant attention in recent years due to its extraordinary physical and chemical properties, ${ }^{1}$ although it is extremely difficult to synthesize defect free graphene at macroscopic scale. Being also graphite-derived, reduced graphene oxide (rGO) appears as an efficient and low-cost solution for the development of large area graphene-based materials. Thanks to the combination of its excellent mechanical properties and chemical tunability, rGO films as well as free-standing rGO paper-like materials are exciting systems for potential applications such as membranes with controlled permeability, anisotropic ionic conductors, mechanically reinforced composites, or transparent, and electrically conductive films. $^{2-4}$

However, the preparation of $\mathrm{rGO}$ from $\mathrm{GO}$ is not trivial. For example, during the chemical reduction of individual GO sheets in aqueous dispersion by hydrazine hydrate to $\mathrm{rGO}$, agglomeration of rGO sheets was observed, making the preparation of homogeneous paper materials from the liquid phase impossible. ${ }^{3,5,6}$ A similar problem with agglomeration of the rGO sheets can be observed during film preparation. Few alternative works have been dedicated to post-reduction processes based on chemical/thermal processes. ${ }^{6-8}$ However, such process can lead to a trade-off between restoring electrical conductivity and maintaining mechanical integrity and flexibility of the papers/films. ${ }^{6}$ Thus, chemical or thermal reduction of GO is necessary to obtain a high value of electrical conductivity but can lead to technical problems during the sample preparation.

a)olena@ua.pt
In the current work, GO solution is used for the preparation of rGO film (by chemical reduction) and rGO paper-like sample (by low-temperature treatment) for further comparative study of their structural, electrical, and thermoelectrical properties.

\section{EXPERIMENT}

A light-brown graphene oxide (GO) solution ( $2 \mathrm{~g} / \mathrm{L})$ was prepared based on the Hummer method. Commercially available natural flake graphite, sulfuric acid (95\% 98\%), potassium permanganate, hydrogen peroxide, and hydrochloric acid were used as received from Sigma-Aldrich. All aqueous solutions were prepared with deionized distilled water. GO was synthesized using the following process: $50 \mathrm{ml}$ of $98 \%$ sulfuric acid was placed in a $250 \mathrm{ml}$ chemical glass flask provided with a magnetic stirrer and fixed in a water bath, with a small amount of added ice. Then $2 \mathrm{~g}$ of graphite powder was added to the acid. After stirring for a few minutes, $6 \mathrm{~g}$ of dry potassium permanganate $\left(\mathrm{KMnO}_{4}\right)$ was added to the reactant mixture. Later, the mixture was heated to $35^{\circ} \mathrm{C}$ and diluted with $100 \mathrm{ml}$ of water during $20 \mathrm{~min}$. The diluted mixture was held for $30 \mathrm{~min}$ at a temperature of $70^{\circ} \mathrm{C}$, followed by addition of $100 \mathrm{ml}$ of water. To decolorize the excess potassium permanganate, $1015 \mathrm{ml}$ of $3 \%$ hydrogen peroxide was poured in. Immediately thereafter, the mixture was passed through a paper filter under evacuation. The filtered out material was mixed with $500 \mathrm{ml}$ of distilled water using a magnetic stirrer for $10 \mathrm{~min}$. The GO fraction was separated from the reaction products using a centrifuge. The final product presented a light-brown GO solution, which did not reveal almost any 
signs of settling under further centrifuging for a prolonged time.

Finally, one part of this GO solution $(100 \mathrm{ml})$ was mixed with hydrazine hydrate $(50 \mathrm{ml})$ and sonicated using a Fisher Scientific FS60 ultrasonic bath cleaner. The obtained solution was dropped on $\mathrm{Si}$ or glass substrates (for electric/thermoelectric measurements) and dried at room temperature. Another part of the GO solution $(200 \mathrm{ml})$ was dried in the Petri dish (diameter $5 \mathrm{~cm}$ ) at as low temperature as $100^{\circ} \mathrm{C}$ during $24 \mathrm{~h}$ and black color paper-like material with size up to $3 \mathrm{~cm}$ was obtained.

Morphology of the studied samples was analyzed by transmission electron microscopy (TEM) (Hitachi 9000) and scanning electron microscopy (SEM) (Hitachi SU-70). The $\mathrm{GO}$ and rGO samples for the TEM characterization were prepared by deposition of a drop of $\mathrm{GO}$ or rGO solution on a holey carbon grid with further drying in air.

$\mathrm{X}$-ray diffraction (XRD) profiles were collected at room temperature in a continuous scanning mode (step 0.02 and time $10 \mathrm{~s}$ ) on Rigaku D/Max-B, Cu K diffractometer in the $2 \theta$ range from $5^{\circ}$ to $36^{\circ}$. The Fourier transform infrared (FTIR) spectra were recorded using a Bruker Tensor 27 FTIR spectrometer, mixing the sample with $\mathrm{KBr}$ (Aldrich, 99\%, FT-IR grade). Raman spectra of analyzed samples were obtained at room temperature in back scattering configuration with a Jobin-Yvon Lab Ram HR using $441.6 \mathrm{~nm}$ (blue) laser line.

Current-voltage (I V) cycles were obtained at room temperature using a Keithley SourceMeter 2400 and two manual probes with tungsten tips. For such typical I V measurements, no special top contacts were deposited on studied samples. Electrical resistivity values were measured by four-point method for square of $1 \mathrm{~cm}^{2}$ in a Van der Paw configuration. ${ }^{9}$

The Seebeck coefficient was obtained using a simple setup system for a parallel (in-plane) measurement. A small temperature difference $\left(<10^{\circ} \mathrm{C}\right)$ was applied between two contacts of the sample, clamping the sample between two high conductance metal blocks. The hot side metal block was heated with an internal heating source, and the cold block was cooled by convection to room temperature. The temperature difference was measured with two platinum temperature sensors (PT100), and the voltage was recorded by high-impedance $(>1 \quad G \Omega)$ voltmeter. The Seebeck

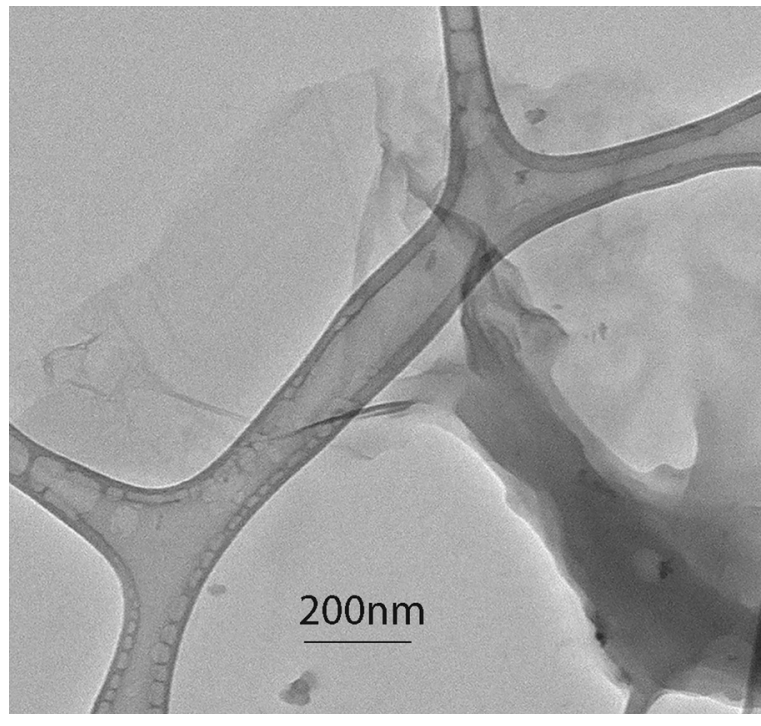

FIG. 1. TEM image of GO.

coefficient was, thus, calculated, dividing the measured voltage by the temperature difference.

\section{RESULTS AND DISCUSSION}

To study the quality of the GO solution, used for further preparation of rGO film and rGO paper analysed in this work, it was investigated in TEM mode. As shown in Figure 1, the continuous layers of GO are confirmed to be rather thin and transparent.

A morphology of the rGO film was observed by TEM and SEM, as shown in Figure 2. TEM image (Fig. 2, left image) indicates that although the rGO film is not atomically flat, it is rather transparent, implying that its thickness is very small. At the same time, the SEM image (Fig. 2, right image) demonstrates a homogeneous microstructure without any visible agglomerations.

SEM images displaying the morphology of the rGO paper are presented in Figure 3. A layered structure is observed with the individual layer thickness of about $2 \mu \mathrm{m}$, resulting in an overall thickness of the paper between $20 \mu \mathrm{m}$ and $30 \mu \mathrm{m}$. The gaps observed between the layers are due to the release of water vapor or $\mathrm{CO}_{2}$ formed during the reduction process. ${ }^{10}$

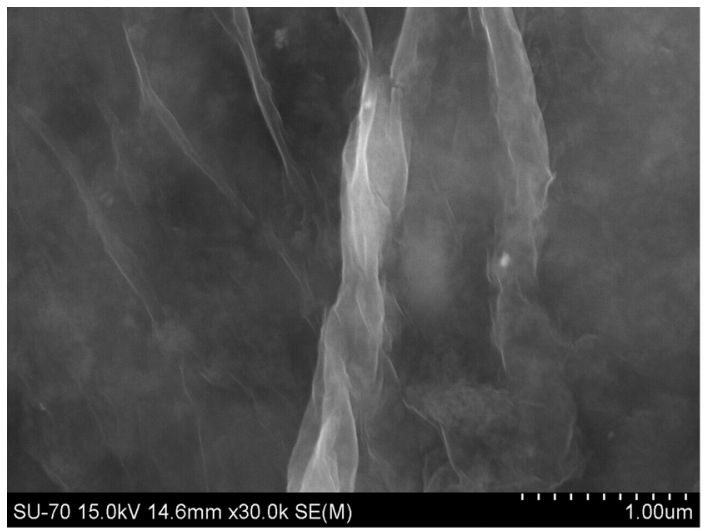

(a)

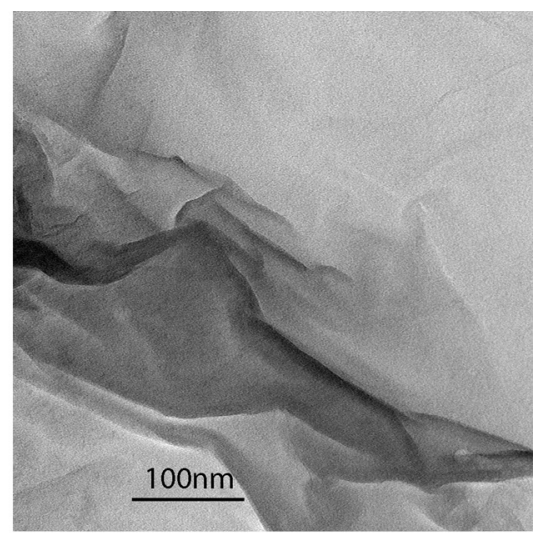

(b)
FIG. 2. TEM (left) and SEM (right) images of rGO film. 


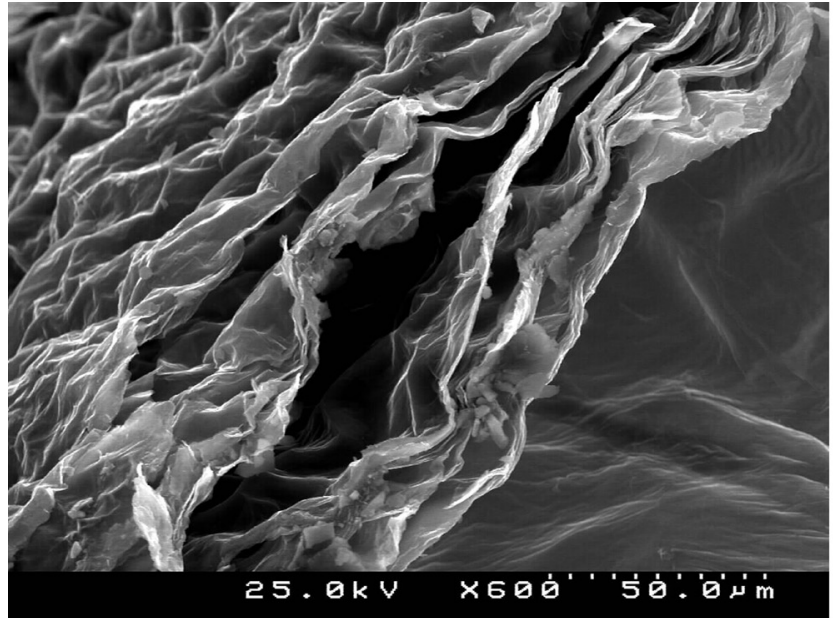

(a)

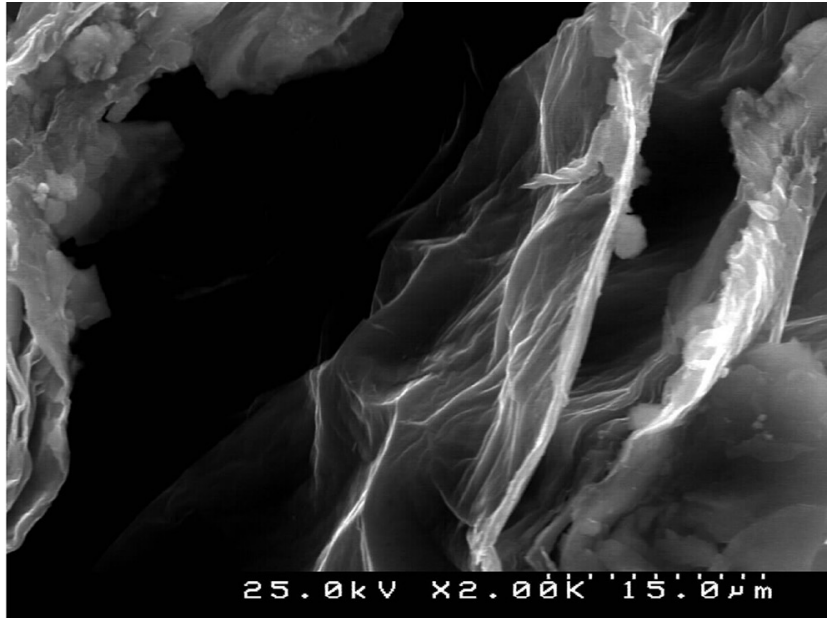

(b)

FIG. 3. SEM images of the rGO paper like material.

XRD profiles of the prepared rGO film and rGO paper material are depicted in Figure 4. A broad peak at $2 \theta$ of about $24^{\circ} 25^{\circ}$ was observed for both samples and ascribed to $\mathrm{rGO}^{11}$ Note this peak cannot correspond to GO, which has a maximum at $2 \theta \sim 10^{\circ} .{ }^{11,12}$ At the same time, a reflection from the pristine graphite can be detected from $26.46^{\circ}$ [Ref. 13] to $30.8^{\circ} .{ }^{14}$ Hence a small peak at about $29^{\circ}$ in the analyzed XRD profile of the rGO paper, which can be detected sometimes in the graphene samples, ${ }^{15}$ is attributed to a weak response from the pristine graphite.

Fourier transform infrared spectra of the analyzed rGO film sample and rGO paper sample are shown in Figure 5. In the case of rGO film, which was reduced by hydrazine hydrate, a small but visible response at $\sim 1060 \mathrm{~cm}^{1}$ was observed and ascribed to the remaining carbonyl groups after the reduction process. ${ }^{16}$ Sometimes after chemical reduction by hydrazine, the stretching vibrations $\mathrm{C} \mathrm{O}$ at $\sim 1060 \mathrm{~cm}^{1}$ are indeed observable and become sharper, being caused by remaining carboxyl groups even after reduction. ${ }^{5}$ However, no chemical reduction was used in the current work for rGO paper sample and hence no band at $\sim 1060 \mathrm{~cm}{ }^{1}$ can be detected in the FTIR spectrum. Instead, a small vibration at $\sim 1380 \mathrm{~cm}{ }^{1}$, similar to that reported in Ref. 16 for rGO film, was found. The peaks observed from $\sim 1060 \mathrm{~cm}{ }^{1}$ to $\sim 1220 \mathrm{~cm}^{1}$ in both spectra of Figure 5 are due to $\mathrm{C}-\mathrm{OH}$ stretching vibrations.

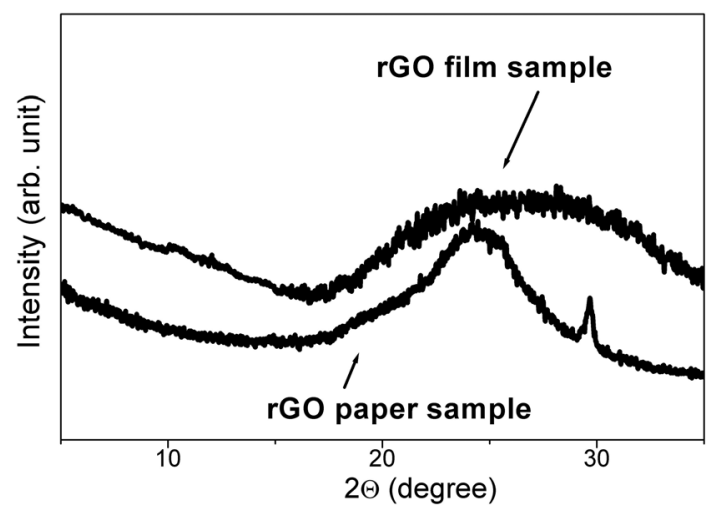

FIG. 4. XRD profiles of rGO film and paper samples.
A skeletal vibration at $\sim 1600 \mathrm{~cm}^{1}$ from unoxidized graphitic domains was observed for both rGO film and rGO paper (at $\sim 1610 \mathrm{~cm}^{1}$ and $\sim 1560 \mathrm{~cm}{ }^{1}$, correspondingly). No stretching vibrations from $\mathrm{C}=\mathrm{O}$ at $\sim 1720 \mathrm{~cm}^{1}$ characteristic for GO spectrum ${ }^{17}$ were detected for the analyzed rGO film and rGO paper. So the FTIR spectra indicate that the oxygen-containing functional groups have been removed. This observation confirms the efficiency of the reduction process of GO in the analyzed rGO film and rGO paper samples.

Raman spectroscopy is a powerful nondestructive technique that is widely used to distinguish order and disorder in the crystal structure of carbon. As usually, the Raman spectrum of graphene consists of several major features: D, G, and $2 \mathrm{D}$ bands. The $\mathrm{G}$ band is related to the in-plane vibration of $\mathrm{sp}^{2}$-bonded carbon atoms and it is the only band coming from a normal first order Raman scattering process in graphene, while the D- and 2D-bands are associated with the vibrations of carbon atoms with $\mathrm{sp}^{3}$ electronic configuration of disordered graphene (originating from a second-order process attributed to local defects, vacancies, and grain boundaries). ${ }^{18}$

The typical Raman spectra of studied rGO-film and paper are shown in Figure 6. They both show the existence of

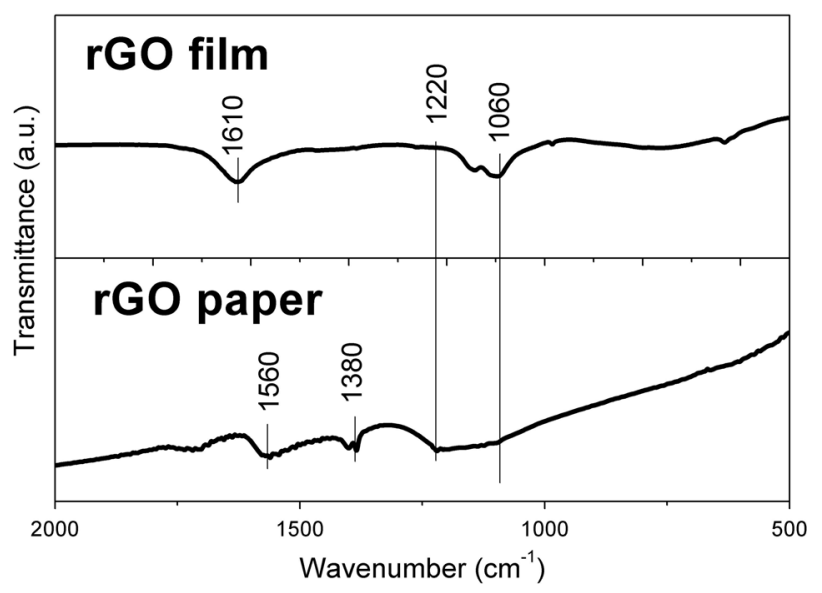

FIG. 5. FTIR spectra of rGO film and paper samples. 


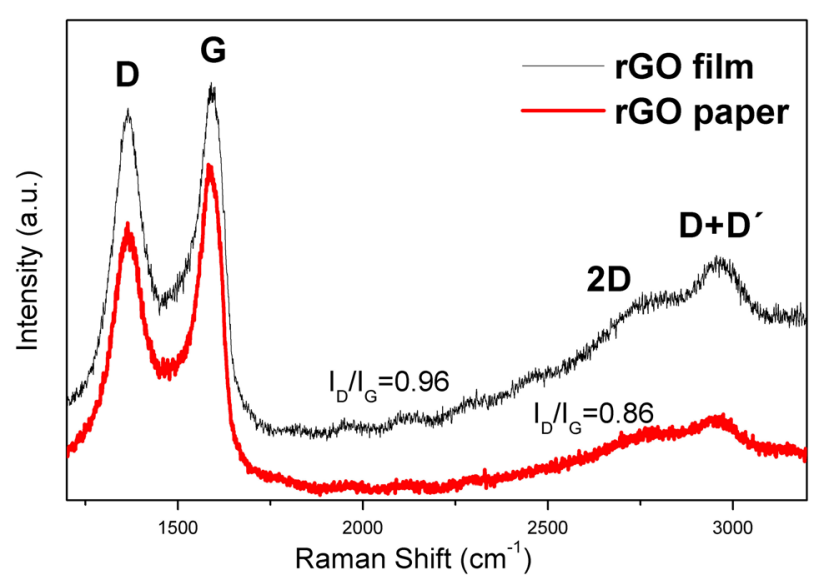

FIG. 6. Raman spectra of rGO film and paper samples.

the $\mathrm{G}$ band (Gaussian peaks fitting data: $\sim 1591 \mathrm{~cm}^{1}$ for film and $\sim 1595 \mathrm{~cm}^{1}$ for paper $)$ and $\mathrm{D}$ band $\left(\sim 1364 \mathrm{~cm}{ }^{1}\right.$ for both film and paper). The intensity ratio of $D$ band to $G$ band $\left(\mathrm{I}_{\mathrm{D}} / \mathrm{I}_{\mathrm{G}}\right)$ is often used as a measure of defect levels in graphitic systems and correlates with the average size of $\mathrm{sp}^{2}$ domains ${ }^{3}$ and the smaller number of $\mathrm{sp}^{2}$ domains leads to the higher ratio $\left(\mathrm{I}_{\mathrm{D}} / \mathrm{I}_{\mathrm{G}}\right)$. In the current work, the intensity ratio $\mathrm{I}_{\mathrm{D}} / \mathrm{I}_{\mathrm{G}}$ of $\mathrm{rGO}$ film $(\sim 0.96)$ prepared with chemical reduction is higher than that for the rGO paper $(\sim 0.86)$ obtained with thermal reduction. This indicates an increasing number of $\mathrm{sp}^{2}$ domains due to the chemical reduction process. ${ }^{3,19}$ Thus, it can be concluded that the rGO-film has been reduced to a slightly higher level than the rGO-paper.

An additional 2D band is usually observed in monolayer graphene samples at $\sim 2680 \mathrm{~cm}^{1}$. Nonetheless, this band cannot be seen in the current samples with as high intensity as would be expected for a pure, defect free graphene. It is well known that Raman shift and shape of the 2D band correlate with the number of graphene layers. ${ }^{20}$ Thus, a broad peak at $\sim 2950 \mathrm{~cm}^{1}$ indicated in Figure 6 as $\mathrm{D}+\mathrm{D}^{\prime}$ can be attributed to a shifted defect-activated vibration of a multilayered material. $^{21}$

I V measurements of rGO film were performed in two cycles as presented in Figure 7, demonstrating a reproducible response. Moreover, the rGO film reveals semiconducting

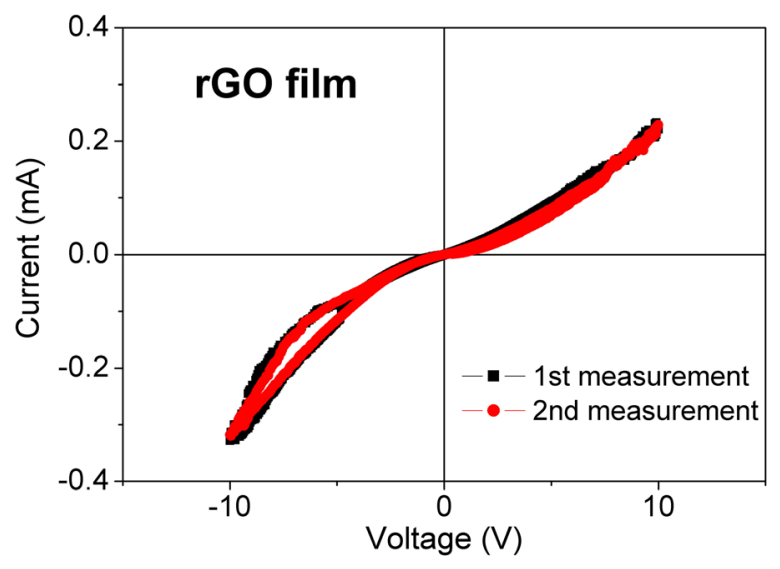

FIG. 7. I V curves of rGO film. behavior with current up to $3 \times 10{ }^{4} \mathrm{~A}$ that is typical for $\mathrm{rGO}$ film. ${ }^{17}$ In contrast, GO films were reported to possess an insulating behavior, revealing high resistance and low current (I $\left.\sim 10{ }^{9} \mathrm{~A}\right) .{ }^{17}$

Figure 8 shows I V curves of analyzed rGO paper-like sample measured in three cycles. During the measurement cycle, the voltage was swept in a sequence of $-1 \mathrm{~V} \rightarrow$ $-10 \mathrm{~V} \rightarrow 0 \mathrm{~V} \rightarrow 1 \mathrm{~V} \rightarrow 10 \mathrm{~V} \rightarrow 0 \mathrm{~V}$ at a rate of $0.01 \mathrm{~V} / \mathrm{s}$. It is seen that during all three measurement cycles, the sample still has high conductivity. Moreover, during the first cycle of the measurement, the current increases dramatically from $10 \mathrm{~mA}$ to $80 \mathrm{~mA}$ at the applied voltage of $\sim-5 \mathrm{~V}$. During the second and third measurement cycles, the peak current is about one order lower but still can be observed between $\pm 3 \mathrm{~V}$ and $\pm 5 \mathrm{~V}$. These reproducible peaks of current induced by voltage pulses of different polarity (bipolar switching) are typical for many materials, including $\mathrm{TiO}_{2}$ [Refs. 22 and 23] or GO. ${ }^{24}$ It can be also seen from Figure 8 that all sweeps present clockwise switching direction at both negative and positive applied voltages, resembling a memristive system of type II. ${ }^{25,26}$ So the I V characteristics of the rGO paper exhibit a typical bipolar switching behavior that corresponds to the memristive system of type II.

From the I V curve in a semilogarithmic scale, shown in inset of Figure 8, it is possible to see that $\mathrm{I} \mathrm{V}$ results are stable and reproducible and currents in the second and third cycles are of order $10^{3} \mathrm{~A}$, being one order higher than that for rGO film. Although this unexpected from the Raman results, which showed that the rGO film is more reduced than the rGO paper, and, hence, should reveal lower resistance and higher currents, it can be explained as follows. The film consists of the graphene flakes and the size of the flakes is very limited, whereas the thickness of the film is very small. Therefore, there can be some discontinuity in the rGO film, making the macroscopic measurements of its electrical properties and precise determination of its electrical parameters very difficult, since the electrical properties of the films can have a contribution from substrates, electrodes, etc. ${ }^{24}$ Such problems are absent in the rGO paper sample.

Indeed, it was not possible to measure the resistivity of the film sample by the four-probe method probably due to discontinuities in the film with very small thickness but large area $\sim 1 \mathrm{~cm}^{2}$. However, the electrical resistivity of the rGO paper measured by the four-probe method was found to be about $1500 \mu \Omega \mathrm{m}$. Correspondingly, electrical conductivity of the rGO paper was calculated as $1 / 1500 \mu \Omega \mathrm{m}=6.7 \times 10^{2} \mathrm{~S} / \mathrm{m}$. This value is three times higher than that reported for a GO paper reduced by hydrazine $\left(1.7 \times 10^{2} \mathrm{~S} / \mathrm{m}\right)$ and about one order lower than that reported for the GO paper obtained by very specific and much more complicated preparation routs, e.g., hydrazine + argon treatment $\left(3.9 \times 10^{3} \mathrm{~S} / \mathrm{m}\right)$, argon treatment $\left(8.1 \times 10^{3} \mathrm{~S} / \mathrm{m}\right)$, and hydrogen treatment $\left(5.0 \times 10^{3} \mathrm{~S} /\right.$ $\mathrm{m}) .{ }^{27}$ In contrast, pure $\mathrm{GO}$ materials contain large $\mathrm{sp}^{2}$ domain sizes that are interrupted by $\mathrm{sp}^{3}$ bonds due to the presence of oxygen, thus showing a very low electrical conductivity of only $8.5 \times 10{ }^{2} \mathrm{~S} / \mathrm{m} .{ }^{27}$ Exposure to vapors of hydrazine makes the GO paper electrically active increasing its conductivity by four orders of magnitude (from $8.5 \times 10^{2} \mathrm{~S} / \mathrm{m}$ to $1.7 \times 10^{2} \mathrm{~S}$ / $\mathrm{m})$ due to the removal of oxygen-containing functional groups 


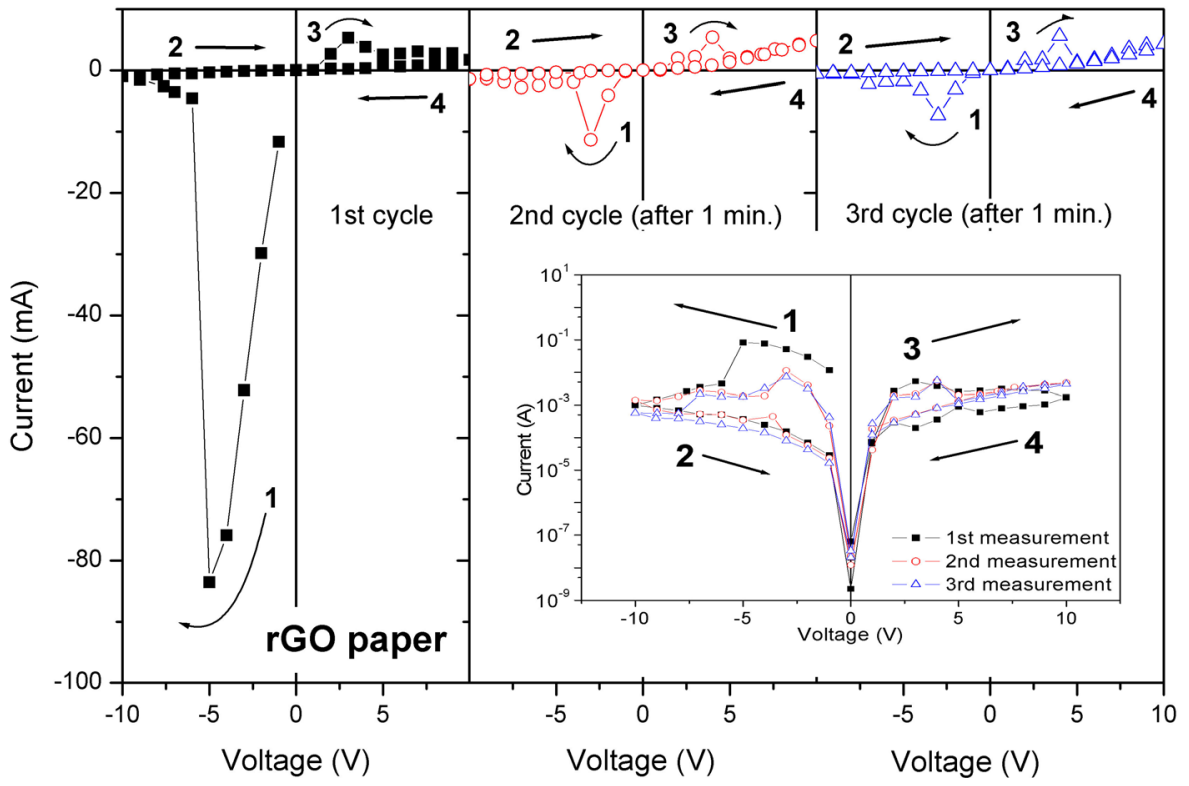

FIG. 8. I V plots of rGO paper like sample during three cycles of measure ment. The inset shows the I V charac teristics in semilogarithmic scale. from the GO paper and enhancement of the connectivity among the graphitic domains by the formation of new $\mathrm{sp}^{2}$ clusters. $^{27}$

Thus, in the current work, rGO paper with a high value of electrical conductivity $\left(6.7 \times 10^{2} \mathrm{~S} / \mathrm{m}\right)$ was obtained by a simple low temperature thermal treatment. It demonstrates that the thermal treatment restores much better $\mathrm{sp}^{2}$ carbon domains in the rGO paper than by chemical reduction, providing a higher reduction degree.

For complete study, thermoelectrical properties of the rGO samples, particularly values of Seebeck coefficient, were measured. However, Seebeck coefficient measurements provided imprecise values for the $\mathrm{rGO}$ film sample probably again due to very small graphene flake size and low thickness of the film and, hence, unwanted contribution of the substrate. At the same time, a Seebeck coefficient of the rGO paper of $-6 \mu \mathrm{V} /$ ${ }^{\circ} \mathrm{C}$ was obtained, indicating that the sample is an n-type semiconductor. A similar absolute value of Seebeck coefficient was found for single layer graphene made by chemical vapor deposition on $\mathrm{Cu}\left(6 \mu \mathrm{V} /{ }^{\circ} \mathrm{C}\right)^{28}$ or for multilayer epitaxial graphene on $\mathrm{Si}\left(-30 \mu \mathrm{V} /{ }^{\circ} \mathrm{C}\right) .{ }^{29}$ It should be stressed here that the Seebeck coefficient can show different values (from $6 \mu \mathrm{V} /{ }^{\circ} \mathrm{C}$ to $180 \mu \mathrm{V} /{ }^{\circ} \mathrm{C}$ ) and depends highly on the method of sample preparation, measurements method, sample thickness, etc. ${ }^{30}$

\section{CONCLUSIONS}

We formed rGO film and rGO paper samples and studied their structural, electrical, and thermoelectric properties. The rGO paper sample obtained by simplified low temperature treatment of the high-quality GO solution at $100{ }^{\circ} \mathrm{C}$ was found to show better electrical properties than the rGO film sample despite the fact that the rGO film was more highly reduced by the chemical route (based on Raman results). From our point of view, this can be explained by discontinuities in the thin film due to the limited graphene flake size and, hence, the presence of the substrate contribution in the rGO film sample electrical response. Moreover, these discontinuities lead to difficulties for macroscopic measurements and precise determination of electrical conductivity and Seebeck coefficient of the rGO film. At the same time, no substrate is needed for the rGO paper sample. Moreover, the thickness of the rGO paper can be easily controlled by the concentration and volume of GO solution. Thus, our approach suggests an easy, costeffective, and environment-friendly fabrication route for conducting graphene paper that is of great potential application as energy storage/harvest (supercapacitors, batteries) and sensors.

\section{ACKNOWLEDGMENTS}

Dr. Antonio Fernandes (i3N, Physic Department, University of Aveiro) is acknowledged for help with Raman measurements. O.O. and G.G. also thank Portuguese Foundation for Science and Technology (FCT) for the Grant Nos. SFRH/BD/77704/2011 and SFRH/BDP/84419/2012, respectively. A.T. acknowledges funds by FEDER through Programa Operacional Factores de Competitividade COMPETE and national funds through FCT within CICECO Project No. FCOMP-01-0124-FEDER-037271 (FCT Ref. PEst-C/CTM/LA0011/2013) and independent researcher Grant No. IF/00602/2013.

${ }^{1}$ K. S. Novoselov, A. K. Geim, S. V. Morozov, D. Jiang, Y. Zhang, S. V. Dubonos, I. V. Grigorieva, and A. A. Firsov, Science 306, 666 (2004).

${ }^{2}$ S. Stankovich, D. A. Dikin, G. H. B. Dommett, K. M. Kohlhaas, E. J. Zimney, E. A. Stach, R. Piner, S. T. Nguyen, and R. S. Ruoff, Nature 442, 282 (2006).

${ }^{3}$ S. Stankovich, D. A. Dikin, R. Piner, K. M. Kohlhaas, A. Kleinhammes, Y. Jia, Y. Wu, S. T. Nguyen, and R. S. Ruoff, Carbon 45, 1558 (2007).

${ }^{4}$ H. Yamaguchi, G. Eda, C. Mattevi, H. K. Kim, and M. Chhowalla, ACS Nano 4, 524 (2010).

${ }^{5}$ D. Li, M. B. Muller, S. Gilje, P. R. Kaner, and G. G. Wallace, Nat. Nanotechnol. 3, 101 (2008).

${ }^{6}$ H. Chen, M. B. Muller, K. J. Gilmore, G. G. Wallace, and D. Li, Adv. Mater. 20, 3557 (2008).

${ }^{7}$ I. K. Moon, J. Lee, R. S. Ruoff, and H. Lee, Nat. Commun. 1, 1 (2010).

${ }^{8}$ O. C. Compton, D. A. Dikin, K. W. Putz, L. C. Brinson, and S. T. Nguyen, Adv. Mater. 22, 892 (2010).

${ }^{9}$ Electrical Measurements, Signal Processing, and Displays, edited by J. G. Webster (CRC Press LLC, 2004).

${ }^{10}$ D. Lin, Y. Liu, Z. Liang, H. W. Lee, J. Sun, H. Wang, K. Yan, J. Xie, and Y. Cui, Nat. Nanotechnol. 11, 626 (2016). 
${ }^{11}$ J. Ding, W. Yan, W. Xie, S. Sun, J. Bao, and C. Gao, Nanoscale 6, 2299 (2014).

${ }^{12}$ J. Che, L. Shen, and Y. Xiao, J. Mater. Chem. 20, 1722 (2010).

${ }^{13}$ E. K. Choi, I. Y. Jeon, S. J. Oh, and J. B. Baek, J. Mater. Chem. 20, $10936(2010)$

${ }^{14}$ Y. Qian, A. Vu, W. Smyrl, and A. Stein, J. Electrochem. Soc. 159, A1135 (2012).

${ }^{15}$ C. M. Willemse, K. Tlhomelang, N. Jahed, P. G. Baker, and E. I. Iwuoha, Sensors 11, 3970 (2011).

${ }^{16}$ Y. Xu, X. Bai, G. Lu, C. Li, and G. Shi, J. Am. Chem. Soc. 130, 5856 (2008).

${ }^{17}$ O. Okhay, R. Krishna, A. Tkach, M. Klaui, L. M. Guerra, J. Ventura, E. Titus, and J. A. Gracio, J. Mater. Sci. 50, 3425 (2015).

${ }^{18}$ F. Tuinstra and J. L. Koenig, J. Chem. Phys. 53, 1126 (1970).

${ }^{19} \mathrm{C}$. Xu, X. Wang, and J. W. Zhu, J. Phys. Chem. C 112, 19841 (2008).

${ }^{20} \mathrm{G}$. Eda and M. Chhowalla, Adv. Mater. 22, 2392 (2010) and references therein.

${ }^{21}$ A. C. Ferrari and J. C. Meyer, Phys. Rev. Lett. 97, 187401 (2006).
${ }^{22}$ H. Mahne, S. Slesazeck, S. Jakschik, I. Dirnstorfer, and T. Mikolajick, Microelectron. Eng. 88, 1148 (2011).

${ }^{23}$ Y. H. Do, J. S. Kwak, J. P. Hong, K. Jung, and H. Im, J. Appl. Phys. 104, 114512 (2008).

${ }^{24}$ S. K. Hong, J. E. Kim, S. O. Kim, and B. J. Cho, J. Appl. Phys. 110, 044506 (2011).

${ }^{25}$ Y. V. Pershin and M. Di Ventra, Adv. Phys. 60, 145 (2011).

${ }^{26}$ D. Biolek, Z. Biolek, and V. Biolkova, Electron. Lett. 47, 1385 (2011).

${ }^{27}$ C. Vallés, J. D. Núnez, A. M. Benito, and W. K. Maser, Carbon 50, 835 (2012).

${ }^{28}$ X. Xu, N. M. Gabor, J. S. Alden, A. M. van der Zande, and P. L. McEuen, Nano Lett. 10, 562 (2010).

${ }^{29}$ A. N. Sidorov, K. Gaskill, M. B. Nardelli, J. L. Tedesco, R. L. Myers Ward, C. R. Eddy, Jr., T. Jayasekera, K. W. Kim, R. Jayasingha, A. Sherehiy, R. Stallard, and G. U. Sumanasekera, J. Appl. Phys. 111, 113706 (2012).

${ }^{30}$ Nanotechnology and Nanomaterials: Advances in Graphene Science, edited by M. Aliofkhazraei (InTech, 2013). 